\title{
Aspectos clínicos e funcionais do paciente com mielomeningocele submetido à cirurgia intraútero
}

\author{
Clinical and Functional Aspects of Meningomyelocele \\ Patient Submitted to Intrauterine Surgery
}

Joyce Xavier Muzzi de Gouvea ${ }^{I}$ Marcela Soares Werneck ${ }^{1}$ Ana Paula Pereira Lemes ${ }^{I}$ Douglas Martins Braga ${ }^{\mathrm{I}}$ ${ }^{\mathrm{I}}$ Associação de Assistência a Criança Deficiente (AACD), São Paulo/ SP - Brasil.
Resumo A mielomeningocele (MMC) desenvolve-se a partir do defeito do fechamento do tubo neural. Afeta os sistemas nervoso, músculo esquelético, urogenital e intestinal, além de apresentar complicações como a hidrocefalia. Os problemas físicos associados incluem graus variados de déficits neurológicos e sensório-motores. $\mathrm{O}$ fechamento da lesão pode ser realizado durante o período pré-natal, por meio da cirurgia intraútero (CIU) ou pós-natal. A CIU tem sido empregada como forma de minimizar complicações neurológicas/motoras, devido à menor exposição do tecido nervoso durante a gestação e no parto. $\mathrm{O}$ tratamento da MMC inclui a atuação multidisciplinar com enfoque no desempenho funcional, sendo que a fisioterapia se concentra na otimização do desenvolvimento neuropsicomotor, promovendo o máximo de independência funcional. O objetivo do presente estudo foi analisar os aspectos clínicos e funcionais do desenvolvimento neuropsicomotor de pacientes com MMC submetidos à cirurgia de correção pré-natal e pós-natal. Foram analisados 143 prontuários de pacientes atendidos no período de março de 2013 a 2018, no setor de fisioterapia aquática da AACD Ibirapuera. Desses, 18 (12,6\%) fizeram a CIU e $125(87,4 \%)$ fizeram a cirurgia após o nascimento. A média de idade dos pacientes foi de 13,1 meses. A idade média do parto foi de 32,72 semanas para os pacientes que fizeram a CIU, evidenciando a prematuridade. Os dados obtidos não mostraram diferenças sobre as etapas do desenvolvimento motor dos pacientes que realizaram a CIU. Sugerimos novos estudos que possam fazer o acompanhamento das etapas do desenvolvimento por um prazo maior.

Palavras-chave: Meningomielocele; Fetoscopia; ReabilitaÇÃO; FISIOTERAPIA

Abstract The Meningomyelocele (MMC), also known as an open spine, develops from the blockage of the neural tube, which begins to develop approximately between 25 and 27 days of gestation. MMC affects the nervous, musculoskeletal, urogenital and intestinal systems, besides presenting complications such as hydrocephalus. Physical problems were associated with neurological and sensory-motor deficits. Neurosurgical blockade of the lesion may be performed during the prenatal period, by intrauterine surgery or postnatal surgery, recommended up to 24 hours after birth. Currently, an MMC bag closure ICU has been used as a form of neurological / motor complications after 
birth due to less exposure of nervous tissue during pregnancy and childbirth. Treatment includes a multidisciplinary action with functional performance, and physical therapy focuses on optimizing neuropsychomotor development, by activating and optimizing preserved muscles, promoting or performing the maximum functional performance. The aim of the present study was to analyze the clinical aspects and the neuropsychomotor development of patients with MMC in prenatal and postnatal correction surgery. We analyzed 143 medical records of patients with MMC who were in therapy from March 2013 to 2018, in aquatic physiotherapy sector of the AACD Ibirapuera. Of the 143 patients, 18 (12.6\%) had intrauterine surgery and 125 (87.4\%) had surgery after birth. The average age of the patients when performing an initial assessment was 13.1 months. The average age of childbirth was 32.72 weeks for intrauterine surgery patients, showing prematurity. There was a lower incidence of ventriculoperitoneal shunt in patients undergoing an intrauterine surgery. The selected data show no differences in the stages of motor development of patients undergoing for intrauterine surgery compared with those who had surgery after birth. We suggest further studies that may follow this developmental stage over a longer period.

Keywords: Meningomyelocele; Fetoscopy; Rehabilitation; Physical Therapy Specialty.

\section{INTRODUÇÃO}

A mielomeningocele (MMC), também conhecida como espinha bífida aberta, desenvolve-se a partir do defeito do fechamento do tubo neural, o qual começa a fundir-se aproximadamente entre o 25 e 27 dias gestacional. ${ }^{1,2}$

O diagnóstico pré-natal pode ser realizado por meio da análise do soro materno (níveis de alfa-feto-proteína AFP), ultrassom de alta resolução e amniocentese. ${ }^{3,4}$

A hidrocefalia torna-se uma consequência importante, pois além da possibilidade de ocasionar déficit cognitivo e motor, dependendo do grau é necessária a colocação de uma válvula de derivação ventrículo-peritoneal (DVP). ${ }^{4}$

A DVP representa um implante de um sistema interno de drenagem com o intuito de retirar excesso de líquor do ventrícu- lo cerebral para a cavidade do peritônio e está relacionada a altos índices de infecção e complicações como mau funcionamento levando à necessidade de troca. ${ }^{2,5}$

O fechamento neurocirúrgico da lesão pode ser realizado durante o período pré-natal, por meio da cirurgia intraútero (CIU) ou pós-natal, preconizada até 24 horas após o nascimento. Atualmente, a CIU de fechamento da bolsa da MMC tem sido empregada como forma de minimizar complicações neurológicas e motoras, após o nascimento, devido à menor exposição do tecido nervoso durante a gestação e no parto. A cirurgia é realizada, geralmente, entre 21 a 27 semanas de gestação, e os critérios são: idade materna e gestacional adequada, gestação única, ausência de anomalias cromossômicas, diagnóstico de hidrocefalia, dilatação ventricular progressiva, entre outros. ${ }^{4,6,7,8}$ 
Os procedimentos neurocirúrgicos fetais podem ser realizados por meio de cirurgias a céu aberto onde é realizada uma incisão na pele da mãe semelhante a uma cesárea, com exposição do útero e retirada do líquido amniótico para correção da lesão. Outra forma de acesso é por meio da fetoscopia que, nesse caso, são introduzidos aparelhos de videolaparoscopia que permitem a visualização por monitores, não sendo necessário cortes no abdome materno. Em ambas as técnicas, a principal complicação é a rotura prematura das membranas. Atualmente, outras formas de abordagem e técnicas vêm sido estudadas a fim de minimizar complicações e aumentar as perspectivas de utilização da abordagem neurocirúgica. ${ }^{5}$

O prognóstico da MMC está relacionado ao nível da lesão, quanto mais alto o nível, mais severa a repercussão. Além disso, espera-se um pior prognóstico se houver complicações associadas, tais como: hidrocefalia, malformação de Arnold-Chiari tipo II sintomática, hidromielia e síndrome da medula presa. ${ }^{1}$

A MMC pode ser classificada em níveis funcionais de acordo com seu comprometimento neurológico: torácico, lombar alto, lombar baixo e sacral., ${ }^{2,9}$

O prognóstico funcional de marcha tem relação direta com o nível da lesão, com a presença ou não de deformidades ortopédicas, hidrocefalia e complicações com a DVP. $^{10,11}$

O tratamento inclui a atuação da equipe multidisciplinar com enfoque no desempenho funcional, sendo fundamental o terapeuta conhecer por qual procedimento de fechamento da bolsa o paciente foi submetido e se houve ou não sequelas após a cirur- gia para traçar os objetivos a serem alcançados e assim definir a melhor conduta a ser seguida. Atualmente já se sabe da importância da intervenção precoce nesses pacientes, dessa maneira, o conhecimento da patologia e prognóstico pela equipe é fundamental para a intervenção mais adequada.

Dentre os tratamentos, a fisioterapia motora se concentra na otimização do desenvolvimento neuropsicomotor, a partir da ativação e otimização da musculatura preservada, promovendo o máximo de independência funcional. , $^{2,11,12}$

\section{Objetivo}

Este trabalho teve como objetivo examinar o perfil funcional dos pacientes com MMC submetidos à cirurgia de correção pré-natal e pós-natal com o intuito de analisar os aspectos clínicos e funcionais do desenvolvimento neuropsicomotor.

\section{MÉTodos}

\section{Sujeitos/procedimento}

Trata-se de um estudo clínico retrospectivo, aprovado pelo Comitê de Ética e Pesquisa da Associação de Assistência à Criança Deficiente (Parecer 3.352.857).

Foram coletados dados dos prontuários dos pacientes, com faixa etária entre 1 e 17 anos, com diagnóstico de MMC, os quais foram atendidos no setor de fisioterapia aquática na AACD Ibirapuera no período de março de 2013 a março de 2018.

Foram incluídos prontuários de pacientes que apresentavam os dados completos: idade materna, fechamento da bolsa intraútero ou pós-natal, idade gestacional do parto, tipo de parto, complicações pós-parto, 
presença de hidrocefalia, presença de DVP, nível motor e etapas do desenvolvimento na avaliação inicial do setor de fisioterapia aquática da AACD Ibirapuera. Foram excluídos prontuários de pacientes incompletos e os que apresentavam outra doença neurológica associada.

\section{Análise estatística}

A normalidade dos dados foi verificada pelo teste de Kolmogorov-Smirnov. Os dados qualitativos foram apresentados em frequência absoluta, relativa e intervalo de confiança de 95\% (por 1000 bootstrapping). Enquanto os dados quantitativos foram descritos por média, mediana, erro padrão, quartis (percentil 25 e 75) e mínimo e máximo. A comparação entre grupos foi realizada por meio do teste de qui-quadrado ou teste Exato de Fisher para as variáveis qualitativas (per- guntas: 'Fez cirurgia intrauterina?, 'Possui DVP' e 'Houve intercorrências com a DVP'). O teste de U de Mann-Whitney foi utilizado para comparar os grupos em relação às variáveis quantitativas. O nível de significância adotado foi um valor de $\mathrm{p}<0,05$. Todas as análises foram realizadas pelo software IBM SPSS $^{\circledast}$ Statistics ${ }^{\circledR}$ versão 21 ou Microsoft Excel.

\section{RESUltados}

Foram pré-selecionados 154 prontuários de pacientes com MMC, 11 foram excluídos (quatro por apresentarem outra patologia neurológica associada e sete por falta de dados no prontuário). 143 prontuários se enquadraram nos parâmetros estabelecidos.

Dos prontuários que apresentaram critérios de elegibilidade, $52,4 \%$ (75) eram do sexo masculino. Dos 143 pacientes, 18 $(12,6 \%)$ fizeram a CIU e $125(87,4 \%)$ fizeram a cirurgia após o nascimento (tabela 1).

Tabela 1 - Estatística descritiva das variáveis qualitativas do estudo

\begin{tabular}{lcccc}
\hline & N & $\%$ & $\begin{array}{r}\text { IC95\% } \\
\text { inferior }\end{array}$ & $\begin{array}{l}\text { IC95\% } \\
\text { Superior }\end{array}$ \\
\hline Sexo & 75 & 52,4 & 44,1 & 60,8 \\
\hline Masculino & 68 & 47,6 & 39,2 & 55,9 \\
Feminino & 143 & 100,0 & 100,0 & 100,0 \\
Total & & & & 92,3 \\
\hline Realizou cirurgia intrauterina (CIU)? & & & & 18,2 \\
\hline Não & 125 & 87,4 & 7,7 & 100,0 \\
Sim & 18 & 12,6 & 100,0 & \\
\hline Total & 143 & 100,0 & & \\
\hline
\end{tabular}

Legenda: IC = intervalo de confiança 
A média de idade dos pacientes ao realizar a avaliação inicial foi de 13,1 meses, sendo de 8,06 meses para os pacientes que realizaram a CIU e de 13,82 meses para os pacientes que realizaram após o nascimento (tabela 2 ).
A idade gestacional média foi de 32,72 semanas para os pacientes que fizeram a CIU e de 37,5 semanas para os que realizaram após o nascimento (tabela 2).

Tabela 2 - Estatística descritiva das variáveis quantitativas do estudo para os grupos de pacientes com e sem cirurgia intrauterina

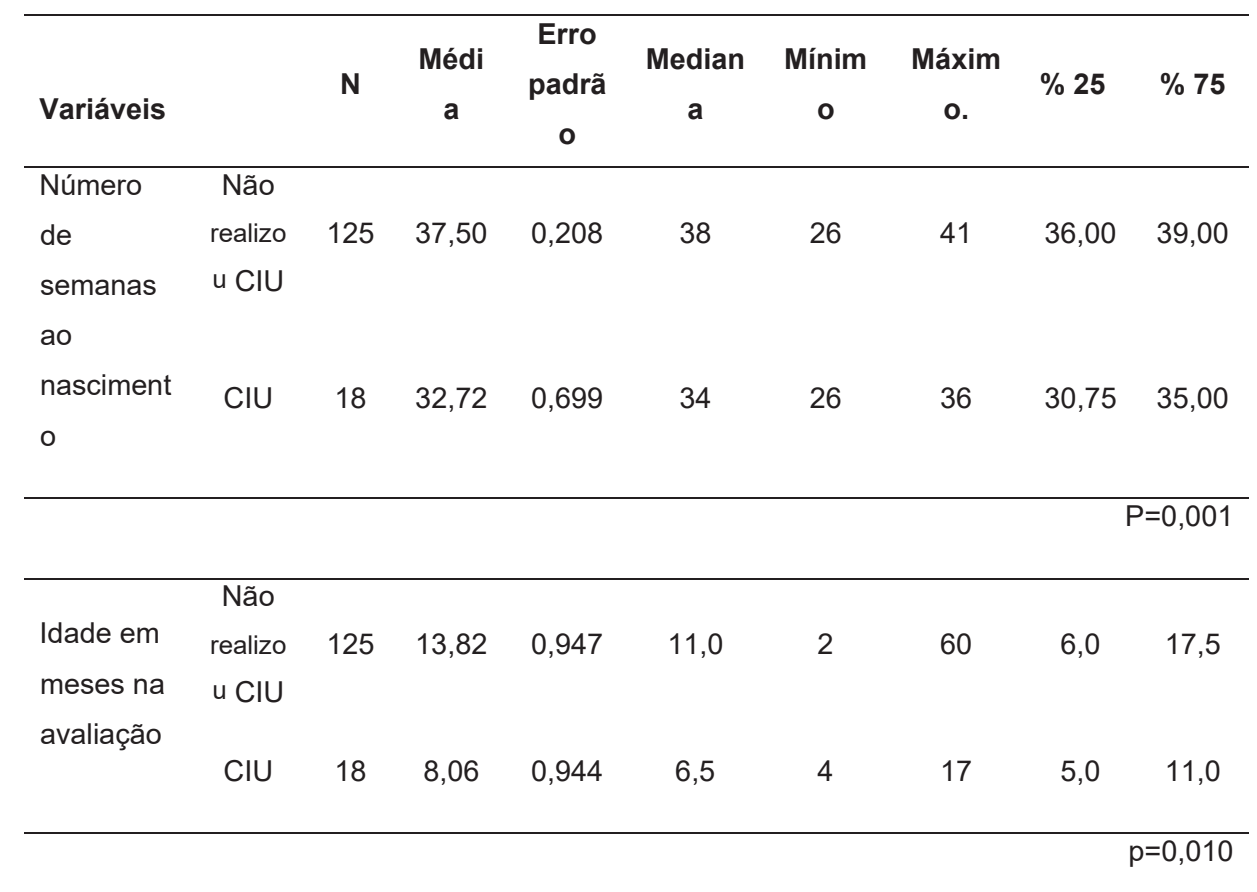

Legenda: CIU = cirurgia intraútero; $\mathrm{N}=$ número de pacientes

Foi observado entre os grupos de pacientes que realizaram a CIU e os pacientes que realizaram a cirurgia após o nascimento (tabela 3) a maior incidência do nível de lesão lombar alto (66\%), sendo que $44,8 \%$ para os pacientes que fizeram a cirurgia após o nascimento e $33,3 \%$ para os que realizaram a CIU.

Em relação à incidência da presença da DVP, 88 (70,4\%) dos pacientes que realizaram a cirurgia após o nascimento apresentavam a DVP; dos pacientes que realizaram a CIU, $5(27,8 \%)$ apresentavam a DVP (tabela 3). A maioria dos pacientes não apresentou intercorrências com a DVP: 66 pacientes (75\%) dos que realizaram a cirurgia após o nascimento e 4 (80\%) que realizaram a CIU (tabela 3 ).

Todos os pacientes do grupo que realizaram a CIU nasceram de cesárea; também se observa essa ocorrência para os pacientes que realizaram a cirurgia após o nascimento, sendo 120 (96\%) no total de cesáreas (tabela 3).

Do grupo que realizou a cirurgia após o nascimento, $88(70,4 \%)$ pacientes tiveram o diagnóstico de MMC durante a gestação (tabela 3). 
Tabela 3 - Estatística descritiva das variáveis qualitativas do estudo para os grupos de pacientes com e sem cirurgia intrauterina

\begin{tabular}{|c|c|c|c|c|c|c|c|}
\hline \multirow{3}{*}{$\begin{array}{c}\text { Realizou cirurgia } \\
\text { intrauterina }\end{array}$} & & & & & & & \\
\hline & \multicolumn{3}{|c|}{ Não } & \multicolumn{3}{|c|}{ Sim } & \multirow{2}{*}{$\begin{array}{l}\chi^{2} \\
\mathbf{P}\end{array}$} \\
\hline & $\mathbf{N}$ & $\%$ & $\begin{array}{l}\mathrm{Cl} 95 \% \text { Cl95\% } \\
\text { inferior superior }\end{array}$ & $\mathbf{N}$ & $\%$ & $\begin{array}{l}\text { IC95\% Cl95\% } \\
\text { inferior superior }\end{array}$ & \\
\hline \multicolumn{8}{|c|}{ Nível neurológico da lesão } \\
\hline TORÁCICO & 25 & 20,0 & $13,627,2$ & 4 & 22,2 & $5,644,4$ & \\
\hline LOMBAR ALTO & 56 & 44,8 & $36,0 \quad 54,4$ & 6 & 33,3 & $11,155,6$ & \\
\hline LOMBAR BAIXO & 21 & 16,8 & $9,624,0$ & 4 & 22,2 & $5,644,3$ & 0,825 \\
\hline SACRAL & 23 & 18,4 & $12,025,6$ & 4 & 22,2 & $5,644,4$ & \\
\hline Total & 125 & 100,0 & $100,0100,0$ & 18 & 100,0 & $100,0100,0$ & \\
\hline \multicolumn{8}{|l|}{ Possui DVP? } \\
\hline Não & 37 & 29,6 & $22,438,4$ & 13 & 72,2 & $50,094,3$ & \\
\hline Sim & 88 & 70,4 & $61,677,6$ & 5 & 27,8 & $5,750,0$ & $<0,001$ \\
\hline Total & 125 & 100,0 & $100,0100,0$ & 18 & 100,0 & $100,0100,0$ & \\
\hline
\end{tabular}

\section{Houve intercorrências com o DVP?}

\begin{tabular}{|c|c|c|c|c|c|c|c|}
\hline Não & 66 & 75,0 & $64,8 \quad 84,1$ & 4 & 80,0 & $40,0100,0$ & \\
\hline Sim & 22 & 25,0 & $15,935,2$ & 1 & 20,0 & $0,060,0$ & $1,00^{*}$ \\
\hline Total & 88 & 100,0 & $100,0100,0$ & 5 & 100,0 & $0,0 \quad 100,0$ & \\
\hline
\end{tabular}

\section{Tipo de parto}

\begin{tabular}{|c|c|c|c|c|c|c|c|}
\hline parto normal & 5 & 4,0 & $0,88,0$ & 0 & 0,0 & $0,00,0$ & \\
\hline Cesárea & 120 & 96,0 & $92,0 \quad 99,2$ & 18 & 100,0 & $100,0 \quad 100,0$ & $1,00^{*}$ \\
\hline Total & 125 & 100,0 & $100,0100,0$ & & & & \\
\hline
\end{tabular}

\section{Diagnóstico de mielomeningocele pré-natal}

\begin{tabular}{|c|c|c|c|c|c|c|c|}
\hline Não & 37 & 29,6 & $22,437,6$ & & & & \\
\hline Sim & 88 & 70,4 & $62,477,6$ & 18 & 100,0 & $100,0100,0$ & \# \\
\hline Total & 125 & 100,0 & $100,0100,0$ & & & & \\
\hline
\end{tabular}

Legenda: IC = intervalo de confiança 
Tabela 4 - Estatística descritiva do desenvolvimento motor para os grupos de pacientes com e sem cirurgia intrauterina

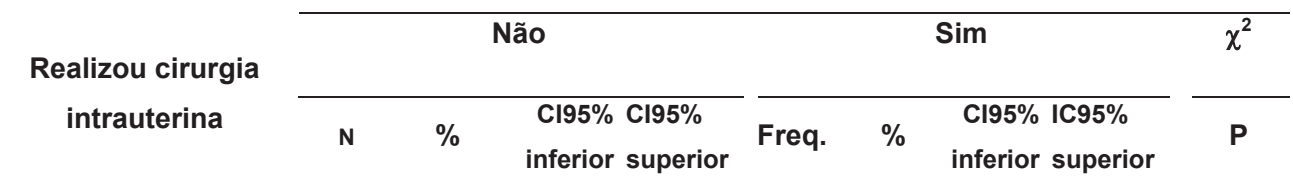

\section{Possui controle cervical?}

\begin{tabular}{lrrrrrrr}
\hline Não & 18 & 14,4 & 8,8 & 20,8 & 6 & 33,3 & $11,155,6$ \\
Sim & 107 & 85,6 & $79,291,2$ & 12 & 66,7 & $44,488,9$ & $0,083^{*}$ \\
Total & 125 & 100,0 & $100,0100,0$ & 18 & 100,0 & $100,0100,0$
\end{tabular}

\section{É capaz de rolar?}

\begin{tabular}{|c|c|c|c|c|c|c|c|}
\hline Não & 56 & 44,8 & $36,0 \quad 54,4$ & 12 & 66,7 & $44,488,7$ & \\
\hline Sim & 69 & 55,2 & $45,6 \quad 64,0$ & 6 & 33,3 & $11,355,6$ & 0,082 \\
\hline Total & 125 & 100,0 & $100,0100,0$ & 18 & 100,0 & $100,0100,0$ & \\
\hline
\end{tabular}

\section{É capaz de se arrastar?}

\begin{tabular}{|c|c|c|c|c|c|c|c|}
\hline Não & 84 & 67,2 & $59,274,4$ & 15 & 83,3 & $66,7 \quad 100,0$ & \\
\hline Sim & 41 & 32,8 & $25,640,8$ & 3 & 16,7 & $0,0 \quad 33,3$ & 0,166 \\
\hline Total & 125 & 100,0 & $100,0 \quad 100,0$ & 18 & 100,0 & $0,0100,0$ & \\
\hline
\end{tabular}

\section{É capaz de sentar-se?}

\begin{tabular}{|c|c|c|c|c|c|c|c|}
\hline Não & 50 & 40,0 & $32,0 \quad 48,0$ & 11 & 61,1 & $38,983,3$ & \\
\hline Sim & 75 & 60,0 & $52,068,0$ & 7 & 38,9 & $16,761,1$ & 0,090 \\
\hline Total & 125 & 100,0 & $100,0 \quad 100,0$ & 18 & 100,0 & $100,0 \quad 100,0$ & \\
\hline
\end{tabular}

Pode ficar em pé com apoio?

\begin{tabular}{cccccccc}
\hline Não & 98 & 78,4 & $71,285,6$ & 16 & 88,9 & $72,2100,0$ & \\
Sim & 27 & 21,6 & $14,428,8$ & 2 & 11,1 & $0,027,8$ & 0,530 \\
Total & 125 & 100,0 & $100,0100,0$ & 18 & 100,0 & $0,0100,0$ & \\
& & & & & & &
\end{tabular}

Os valores de $\mathrm{p}$ correspondem ao teste de qui-quadrado, exceto quando assinalados com ${ }^{\star}$, que indica a utilização do teste Exato de Fischer. 
Na tabela 4 estão os dados do desenvolvimento motor dos pacientes do grupo que realizou a CIU e do grupo que realizou a cirurgia após o parto. Não houve diferença estatisticamente significante entre os grupos estudados.

Podemos observar que houve uma grande variabilidade de idade entre os sujeitos da pesquisa, sendo assim, pode-se considerar que algumas habilidades motoras podem não ser esperadas para determinados sujeitos. Dessa forma, é importante salientar que a idade do indivíduo é fundamental para saber o que esperar ou não das etapas do desenvolvimento neuropsicomotor e assim poder avaliar um possível atraso motor.

\section{Discuss Ão}

A MMC é um dos mais complexos defeitos congênitos e os cuidados exigem atendimento multidisciplinar às suas disfunções, desde a atenção às complicações como hidrocefalia, bexiga e intestinos neurogênicos, mobilidade e déficits cognitivos, até a promoção da saúde. . $^{1,3}$

O fechamento cirúrgico da bolsa da mielomeningocele é uma etapa fundamental e pode ser realizado durante o período prénatal ou pós-natal, preconizada até 24 horas após o nascimento. ${ }^{4,5,6}$

Neste estudo, os pacientes com MMC foram igualmente do sexo feminino e masculino, mais frequentemente com lesão de nível lombar alto tanto para os pacientes que fizeram a cirurgia após o nascimento quanto para os que realizaram a CIU, esses dados vão de encontro com a literatura que também não observou uma prevalência a respeito da frequência entre os sexos. ${ }^{7,8}$
Danzer e Adzick (2011) ${ }^{13}$ observaram que a CIU está relacionada com um aumento do parto prematuro. Verificamos em nosso estudo que a média do número de semanas de gestação foi de 32,72 semanas para os pacientes que fizeram a CIU, evidenciando uma prematuridade nesses pacientes.

Observou-se uma maior incidência da presença da DVP nos pacientes ( $\mathrm{n}=88$ $70,4 \%$ ) que realizaram a cirurgia após o nascimento; dos pacientes que realizaram a CIU, $5(27,8 \%)$ apresentavam a DVP $(<0,001)$. Bevilacqua e Pedreira (2015) ${ }^{14}$ descrevem que há uma redução da incidência da DVP em pacientes que realizaram a CIU. Danzer e Adzick (2011) ${ }^{13}$ observaram uma redução significativa no risco da realização da DVP com 1 ano de idade, mas sugerem novos estudos que acompanhem esses resultados ao longo dos anos. Kabagambe e colaboradores $(2018)^{6}$ também relatam a redução da realização da DVP quando o paciente atinge 1 ano de vida.

Todos os pacientes do grupo que realizaram a CIU nasceram de cesárea; também se observou essa maior ocorrência para os pacientes que realizaram a cirurgia após o nascimento sendo 120 (96\%) no total de cesáreas. O alto índice de cesáreas se justifica visto que quando realizadas por motivos médicos, as cesarianas podem reduzir a mortalidade e morbidade materna e perinatal (OMS, 2015) $)^{15}$.

Os dados obtidos não mostraram diferenças sobre as etapas do desenvolvimento motor dos pacientes que realizaram a CIU e do grupo que realizou a cirurgia após o parto. Bevilacqua e Pedreira $(2015)^{14}$ relatam que a realização da CIU evita a progressão da lesão e permite um desenvolvimen- 
to nervoso mais próximo da normalidade; além disso, em seu estudo, os pacientes submetidos à CIU apresentaram melhores resultados, como aumento de duas vezes da probabilidade de deambulação em relação aos pacientes que realizaram a cirurgia após o nascimento. Kabagambe e colaboradores $(2018)^{6}$ descrevem uma melhora para o desenvolvimento mental e função motora, para pacientes que realizaram a CIU, aos 30 meses de idade.

Visto que os dados do desenvolvimento motor dos pacientes foram colhidos na avaliação inicial e a média de idade foi de 13,1 meses (sendo de 8,06 meses para os pacientes que realizaram a CIU e de 13,82 meses para os pacientes que realizaram após o nascimento), é possível que os pacientes tivessem um avanço nas etapas do desenvolvimento ao longo dos meses, após o início do processo de reabilitação. Sugerimos novos estudos que possam fazer esse acompanhamento em longo prazo.

\section{Conclusão}

Os dados deste estudo evidenciaram uma prematuridade para os pacientes que realizaram a CIU, mas uma menor incidência da presença da DVP nesses pacientes. Os dados obtidos não mostraram diferenças sobre as etapas do desenvolvimento motor dos pacientes que realizaram a CIU em comparação com aqueles que fizeram a cirurgia após o nascimento. Sugerimos novos estudos que possam fazer esse acompanhamento das etapas do desenvolvimento por um prazo maior.

\section{REFERÊNCIAS}

1. Bizzi JWJ, Machado A. Mielomeningocele: conceitos básicos e avanços recentes. jbnc. 2012; 23(2):138-51.

2. Scontri CMCB, Braga D, Gôuvea JXM; Werneck MS. Associação entre objetivo funcional e nível de lesão na Mielomeningocele. Revista CIF Brasil. 2019; 11(1):17-31

3. Liptak GS, Dosa NP. Myelomeningocele. Pediatrics in Review. 2010; 31(11):443-450

4. Silva MAL, Carvalho R. Atuação no intraoperatório da correção cirúrgica de mielomeningocele a "céu aberto" intraútero. Revista SOBECC. 2015; 20(2):113-118.

5. Zanon N, Grecco LAC, Caselato GCR, Pedreira D. Neurocirurgia Fetal - atualidades e perspectivas. Rev. bras. neurol. psiquiatr. 2014; 18(2): 130-8

6. Kabagambe SK, Jensen GW, Chen YJ, Vanover MA, Farmer DL. Fetal Surgery for Myelomeningocele: A Systematic Review and Meta-Analysis of Outcomes in Fetoscopic versus Open Repair. Fetal Diagn Ther. 2018; 43(3):161-74

7. Ulsenheimer MMM, Antoniuk SA, Santos LHC, Ceccatto MP, Silveira AE, Ruiz AP, et al. Myelomeningocele - a brazilian university hospital experience. Arq. Neuro-Psiquiatr. 2004; 62(4): 963-8

8. Collange LA, Franco RC, Esteves RN, Zanon-Collange N. Desempenho funcional de crianças com mielomeningocele. Fisioter. Pesqui. 2008; 15(1): 58-63

9. Argento-Giacoletti A, Warschausky SA, Shank L, Hornyak JE.. "Spina Bifida Myelomeningocele". In: Goldstein S, Reynolds C. R. Handbook of neurodevelopmental and genetic disorders in children. New York: Guilford Press; 2011.

10. Flanagan A, Gorzkowski M, Altiok H, Hassani S, Ahn KW. Activity level, functional health, and quality of life of children with myelomeningocele as perceived by parents. Clin Orthop Relat Res. 2011; 469(5):1230-5. 
11. Bartonek A. Motor development toward ambulation in preschool children with myelomeningocele--a prospective study. Pediatr Phys Ther. 2010;22(1):52-60

12. Marinho-Buzelli AR, Bonnyman AM, Verrier MC. The effects of aquatic therapy on mobility of individuals with neurological diseases: a systematic review. Clin Rehabil. 2015; 29(8):741-51

13. Danzer E, Adzick NS. Fetal surgery for myelomeningocele: patient selection, perioperative management and outcomes. Fetal Diagn Ther. 2011;30(3):163-73.

14. Bevilacqua NS, Pedreira DAL. Cirurgia fetal endoscópica para correção de mielomeningocele: passado, presente e futuro. einstein. 2015;13(2):283-9

15. ORGANIZAÇÃO MUNDIAL DA SAÚDE. Declaração da OMS sobre Taxas de Cesáreas. 2015. Disponível em: https://apps.who.int/iris/bitstream/handle/10665/161442/WHO_RHR_15.02_ por.pdf;jsessionid=7E1729F3D96E3A8E6EF118E64EDC3A67? sequence=3. Acesso em: 10 nov. 2019.

\section{DADOS DOS AUTORES}

\section{MARCEla SoARes Werneck}

Fisioterapeuta do setor de fisioterapia aquática da Associação de Assistência a Criança Deficiente (AACD), São Paulo/SP - Brasil. mwerneck@aacd.org.br

\section{Joyce Xavier Muzzi de Gouvea}

Fisioterapeuta do setor de fisioterapia aquática da Associação de Assistência a Criança Deficiente (AACD), São Paulo/SP - Brasil. jgouvea@aacd.org.br

\section{Ana Paula Pereira lemes}

Aluna do curso de aperfeiçoamento em fisioterapia, na área de reabilitação física, com enfoque interdisciplinar da Associação de Assistência a Criança Deficiente (AACD), São Paulo/SP - Brasil. anapaulah.s2@hotmail.com

\section{Douglas Martins Braga}

Coordenador do setor de fisioterapia aquática da Associação de Assistência a Criança Deficiente (AACD), São Paulo/SP - Brasil. dbraga@aacd.org.br/douglasbraga78@hotmail.com

Submetido em: 16-9-2020

Aceito em: 9-6-2021 